\title{
Expanding Capabilities of Low-kV STEM Imaging and Transmission Electron Diffraction in FIB/SEM Systems
}

\author{
Tomáš Vystavěl ${ }^{1}$, Lubomír Tůma ${ }^{1}$, Pavel Stejskal ${ }^{1}$, Marek Unčovský ${ }^{1}$, Jan Skalický $^{1}$, Richard Young ${ }^{2}$ \\ 1. Thermo Fisher Scientific, Vlastimila Pecha 12, Brno, Czech Republic \\ 2. Thermo Fisher Scientific, 5350 NE Dawson Creek Drive, Hillsboro, OR, USA
}

High resolution low-kV STEM imaging is getting more and more attention in the materials research and semiconductor industry, as well as in life sciences research [1]. This has been driven by the need to work with thinner TEM lamella, and with samples containing low-Z and beam sensitive materials. Decreasing electron energies is often favorable from a radiation damage point of view, moreover it improves scattering contrast. In this contribution we focus on STEM imaging extended by diffraction analysis, by integration of pixelated detectors into the FIB/SEM platform.

There are two main types of STEM instrumentation - dedicated S/TEM systems (which can typically perform in both STEM and TEM modes) and general purpose FIB/SEM systems with a STEM option. The dedicated S/TEM instruments offer higher ultimate imaging performance, usually can go to higher accelerating voltages, might use correctors and advanced analytical techniques such as EELS. In contrast, FIB/SEM platforms offer high system versatility, combining sample preparation with imaging and analysis. However, the STEM options have historically been limited to $\sim 6 \AA$ or more, limiting their usefulness on the most challenging samples. Significant improvements in this field was achieved by introducing the Helios G4 platform which brings $3 \AA$ STEM resolution, as well in-situ site specific ultrathin lamellas with damage below $1 \mathrm{~nm}$ and maximum cut fidelity [2].

Resolution of a $30 \mathrm{kV}$ field emission SEM is mainly limited by spherical aberration of the objective lens. The easiest way how to reduce the spherical aberration is to shorten the focal length of the objective lens. In SEMs with conventional lenses the minimum focal length is usually more than $10 \mathrm{~mm}$, in instruments with immersion magnetic (single pole) lenses more than $4 \mathrm{~mm}$. On the other hand, in TEMs where the objective lens utilizes two pole pieces with small gap in between (an in-lens configuration), the focal length can be several times shorter and consequently the coefficients of axial aberrations smaller. The presented accomplishment of STEM performance improvement in a SEM is to equip the system with a second (lower) pole piece in such a way that the system is in-situ configurable and can be operated both in a traditional single-pole SEM (or FIB/SEM) mode and in a high resolution in-lens STEM mode. In order to have the system user-configurable the lower pole piece with integrated multi-segment solid state detector is mounted on a retractable mechanism, which also assures precise mechanical alignment to avoid unwanted aberrations. Improvements in resolution at $30 \mathrm{kV}$ was demonstrated by lattice planes visualizations on several materials such as carbon nanotubes $(0.34 \mathrm{~nm})$, silicon $(0.31 \mathrm{~nm})$ or tungsten disulfide $(0.27 \mathrm{~nm})$ as shown in Figure 1c.

To augment the STEM imaging it is desirable to view the diffraction pattern from the sample (as would typically be the case in a conventional S/TEM), which can provide information about sample crystallography, as well as lamella orientation. To investigate this, a pixelated detector based on the Medipix detector family [3] was used. Results are shown in Figures 1c and 2b. The acquired diffraction pattern images have a very high dynamic range, achieved by the ability to count every single electron in the digital domain and the resulting noise-less operation. 


\section{References:}

[1] Kaiser, U, Stöger-Pollach, M, Ultramicroscopy, 145, (2014), p.1.

[2] Vystavel et al, Microscopy and Microanalysis, 22(S3), (2016), p. 30.

[3] http://medipix.web.cern.ch/medipix/

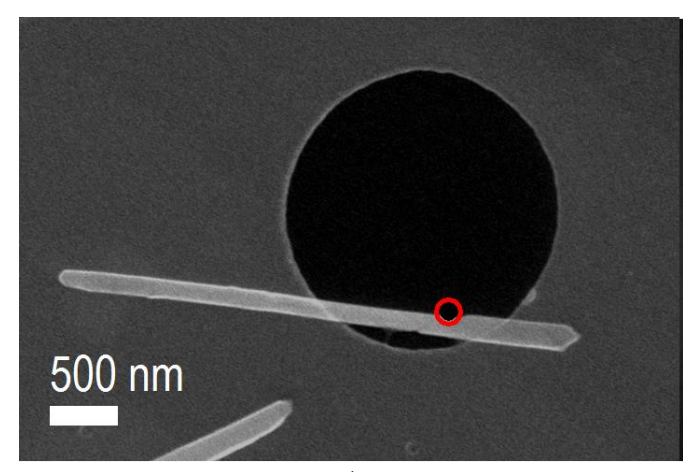

a)
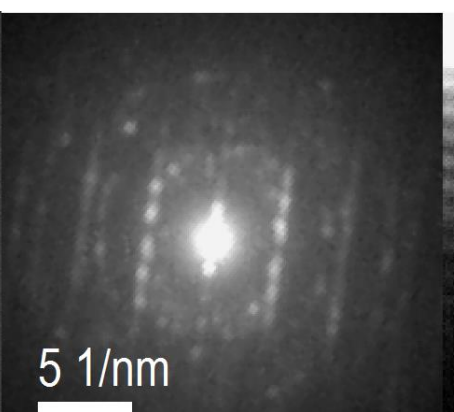

\section{$2 \mathrm{~nm}$}

Figure 1. Helios G4 imaging of tungsten disulfide at $30 \mathrm{kV}$ showing (a) secondary electron image, (b) corresponding $30 \mathrm{kV}$ nano-diffraction acquired on a Medipix detector and (c) bright field STEM image revealing planes with $6.2 \AA$ and $2.7 \AA$ spacing respectively.

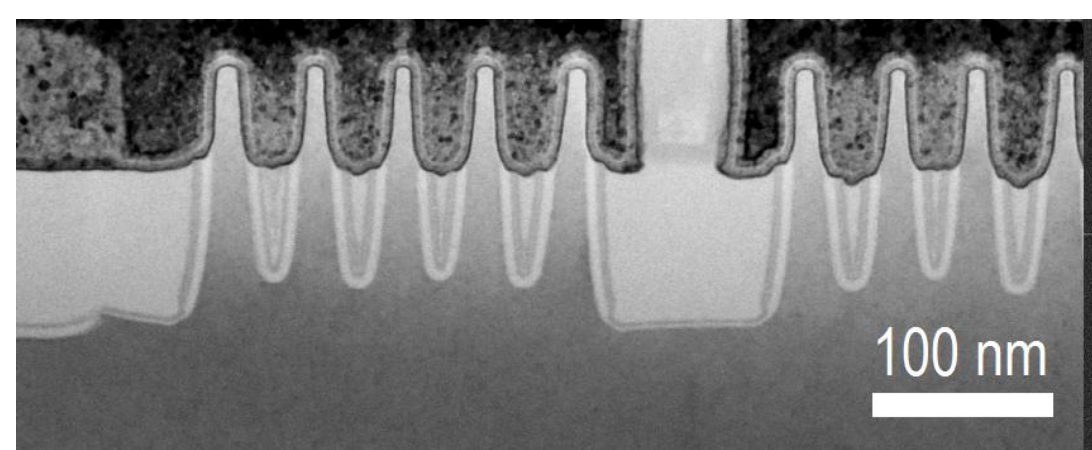

a)

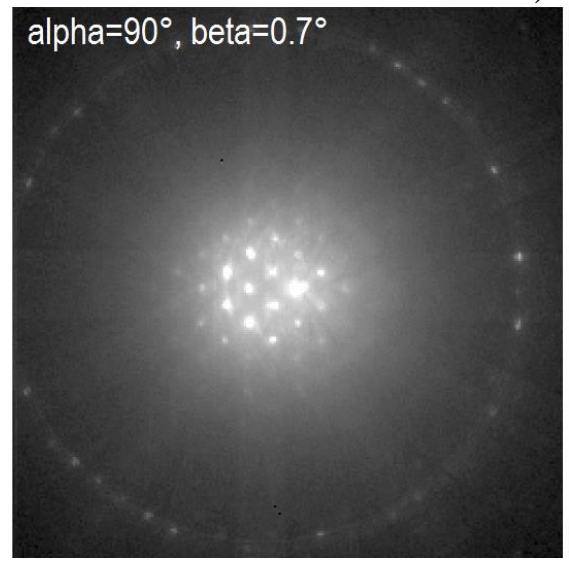

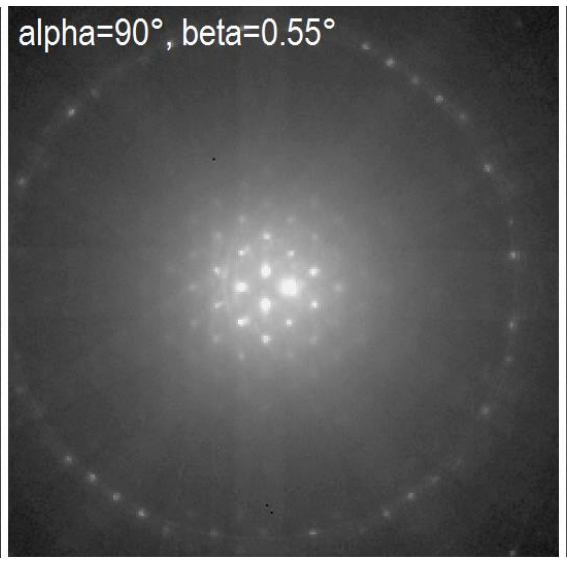

c)

Figure 2. (a) typical $30 \mathrm{kV}$ STEM image of a $14 \mathrm{~nm}$ semiconductor device finished with low $\mathrm{kV}$ ion beam cleaning, substrate at [100] zone axis, (b) FFT from silicon substrate region, taken from higher magnification picture, $\{111\}$ planes are resolved, (c) sequence of diffraction patterns acquired during sample alignment to the [100] zone axis orientation, tilt angles of double tilt rod are marked on insets. 